\title{
Typenbildung: Internationale Erfahrungen und neue europäische Fragen
}

\section{Zugleich eine Besprechung zu Christoph A. Kern, Typizität als Strukturprinzip des Privatrechts. Ein Beitrag zur Standardisierung übertragbarer Güter, Tübingen (Mohr Siebeck) 2013, XLI, 614 S., 109,- € ISBN 978-3-16-151724-2 \\ Christian Baldus*}

1. Die Zweischneidigkeit des Typus ist bekannt: Er kann einerseits Vorstufe der Normbildung, also „harter“ Kategorienbildung, sein, und dann möglicherweise sachgerecht (Beispiel: reflektierte Strukturierung von Kasuistik bei vergleichbaren Interessenlagen). Andererseits kann er der Auflösung existierender harter Kategorien dienen, was man nicht sachgerecht finden sollte (anders bekanntlich viele Stimmen vor 1945 und einige auch später). Evident sachwidrige Phänomene sind bekannt (Beispiel: Lehre vom „Tätertyp“).

Diese Zweischneidigkeit und Missbrauchsanfälligkeit erklärt sich auch daraus, dass die Begriffsdiskussion noch jung ist. Savigny kennt den Typus im „System“ zwar. Dort erscheint er jedoch etwas verloren zwischen Kernbegriffen (und so findet der Begriff sich bezeichnenderweise nicht im Register der 2. Auflage von 1856): ${ }^{1}$ Die Rechtsregel, deren „Ausprägung im Gesetz“ und das Rechtsverhältnis (also Savignys Zentralkategorie) leiten sich vom („organischen“) Rechtsinstitut ab; Savigny lehrt, dass nach dem „Wesen der Sache“ „in der That jedes Rechtsverhältnis unter einem entsprechenden Rechtsinstitut, als seinem Typus, steht, und von diesem auf gleiche Weise beherrscht wird, wie das einzelne Rechtsurtheil von der Rechtsregel“. ${ }^{2}$ Zum Typus sagt er an dieser Stelle weiter nichts, sondern geht zur systematischen Verknüpfung der Institute über. Ohne Wurzeln und Fortführung des Gedankens hier untersuchen zu können: Für Savigny ist der Typus an dieser Stelle ein bloßer Begleitbegriff. Dessen spätere Karriere fand in einem pandektistisch nicht vollständig geordneten Begriffsraum und gegen das pandektistische Anliegen statt, belastbare Kategorien der Rechtsanwendung zu schaffen.

Und doch gebrauchen wir den Typus gerade in Gebieten, die geradezu paradigmatisch mit Begriff und Sicherheit verbunden sind - wie dem Sachenrecht. Wer „Typizität“ statt „Typus“ sagt, setzt sich damit möglicherweise bereits sprachlich vom zersetzenden Aspekt der Typisierung ab. Das Interesse einer privatrechtswissenschaftlichen Betrachtung muss also darauf gerichtet sein, auch im Lichte historischen Gebrauchs und Missbrauchs die Leistungsfähigkeit von Typus und Typizität zu bestimmen.

* Prof. Dr. Christian Baldus lehrt Bürgerliches Recht und Römisches Recht, Europarecht und Rechtsvergleichung an der Universität Heidelberg.

1 Das Register ist von Heuser, einem Praktiker, der in diesem Stichwort anscheinend keinen Gewinn sah.

2 Friedrich Carl von Savigny, System des heutigen römischen Rechts, I (Berlin 1840) 9f. Kursive: C.B. 
Im Folgenden kann und soll selbstverständlich keine Typologie der Typologien gegeben werden. Auch die - um es vorwegzunehmen: vorzügliche - Arbeit von Kern kann nicht in allen Punkten dargestellt werden. Wohl aber ist der Diskussionsstand in den klassischen Feldern des Zivilrechts zu skizzieren, denn das hier zu besprechende Werk setzt eines dieser Felder - das Sachenrecht - in Beziehung zu einem im Ansatz klassischen, aber höchst dynamischen Gebiet des Wirtschaftsrechts, nämlich zum Wertpapierrecht; dies unter der Leitfrage, wie sich Typizität strukturell zu Information, privatrechtlich also: Publizität verhält.

2. Vor allem aus rechtsökonomischer Sicht ist vorgetragen worden, zentral gehe es heute um Informationszugang, namentlich in „modernen“ Rechtsgebieten. In einer solchen Perspektive wird Publizität zu einem Sonderfall und Typizität zum Relikt. $\mathrm{Ob}$ sie aber auf richtigen Voraussetzungen beruht, ist gerade im Lichte ökonomischen Denkens zu fragen: Den optimal informierten und auch noch optimal entscheidenden Wirtschaftsteilnehmer gibt es bekanntlich nicht. Dem Recht ist aber mit bloßen Modellfiguren nicht gedient. Die Frage lautet also, ob typenbasierte Ansätze den realen Regelungsaufgaben des Rechts möglicherweise eher gerecht werden. Kern fragt praxisgerecht und systembewusst nach Standardisierbarkeit. Schon aus seinem Ansatz wird deutlich, dass er weiß, was gern vergessen wird: Modernität, auch Modernität wissenschaftlichen Arbeitens, ist keine Frage des Objekts. Wissenschaften definieren sich primär nicht durch ihre Gegenstände, sondern durch ihre Erkenntnisinteressen und Methoden - Regenwurmrecht ist keine Zoologie, Computerrecht keine Informatik. Kern trägt also einen bewährten Rechtswissenschaftsbegriff an sein Sujet heran. Schauen wir aber zunächst noch einmal auf den titelgebenden Begriff und dessen Anwendungsfelder.

3. In der Rechtswissenschaft wird heute erstaunlich selten über den Sinn und Unsinn von Typenbildung gesprochen, obwohl die Praxis ständig mit Typen arbeitet. Dies betrifft sowohl das Obligationenrecht (unten 4.) als auch das Sachenrecht; im Erbrecht fehlt eine Typendiskussion so gut wie ganz (5.), obwohl es praktische Fragen gibt, bei denen man sie führen könnte (etwa in Anlehnung an die $\mathbb{S} \$ 1937-1941$ ), ${ }^{3}$ und obwohl eine solche Diskussion im Überschneidungsfeld von Sachenrecht und Erbrecht alsbald zu erwarten ist (näher unten 6.); schließlich lassen sich auch bestimmte das Familienrecht betreffende Fragen möglicherweise als Typenfragen fassen (7.). In dieser Lage klärt Kerns Habilitationsschrift, sinnfällig angelegt und strukturiert (8.), ihren unmittelbaren Themenbereich eindrucksvoll (9.); sie erlaubt zugleich weiterführende historische (10.) und systematische (11.) Erwägungen zur Leistungsfähigkeit des Typenbegriffs - dies nicht zuletzt deswegen, weil die Arbeit ein Musterbeispiel historisch unterlegter und systematisch angelegter Rechtsverglei- 
chung ist. Sie zeigt damit, was Dogmatik unter den Bedingungen der Internationalisierung und Ökonomisierung des Rechts leisten kann (12.). ${ }^{4}$

4. Im Schuldrecht (vgl. auch Kern S. 537-540), wo die Typen sich wohl am stärksten verändern, behilft man sich in erster Linie mit dem Hinweis auf die Privatautonomie. In zweiter Linie sucht man die Grundsatzdiskussion über das Verhältnis zwischen dispositivem Recht und ergänzender Vertragsauslegung zu vermeiden. In dritter nimmt man es einstweilen hin, dass bestimmte Vertragstypen des BGB jedenfalls nach hundert Jahren einer Neuformulierung bedürften; die sinnvollen Ansätze der Schuldrechtsreform harren auch insoweit einer Fortführung. Immerhin ist der Behandlungsvertrag jetzt kodifiziert, ${ }^{5}$ und zu bestimmten Sonderformen des Werkvertrages wird diskutiert, ob sie eines eigenen Typus bedürfen.

Diese Entwicklungen betreffen nicht zufällig den Bereich der, weit und untechnisch gesprochen, Dienstleistungen: Jedenfalls in ökonomischer Perspektive haben die unkörperlichen Leistungen gegenüber dem Kauf an Bedeutung gewonnen, was seit langem wissenschaftliches Interesse weckt. Unklar ist freilich, was aus diesem Bedeutungsgewinn für die Prägekraft des einen oder anderen Vertragstyps namentlich in gesetzgeberischer Hinsicht folgt (aber auch an Analogien ist zu denken, wie nach der Schuldrechtsreform gesehen). ${ }^{6}$ Das Unionsrecht tendiert dazu, mit einem Binom „Güter und Dienstleistungen“ das Problem zu umschiffen; ${ }^{7}$ jüngere Reformversuche im europäischen Schuldrecht scheinen den Kauf nach wie vor für das Paradigma des Schuldvertrages zu halten, wogegen aus historischer, vergleichender und praktischer Sicht einige Bedenken bestehen: Kaum ein Vertragstyp ist derart unübersichtlich und uneinheitlich geregelt wie der Kauf, was natürlich mit seinem Verhältnis zum Eigentum zusammenhängt, aber auch damit, dass Verbraucherrecht hier besonders früh systemverändernde Wirkung entfalten konnte.

Die mittlerweile entstandene Debatte um bestimmte typenbezogene Instrumente und Vorschläge im Unionsrecht konzentriert sich einerseits auf technische Mängel etwa

4 Diese Frage ist bekanntlich ein zentrales Anliegen der Schule, aus welcher die Arbeit kommt; dazu am Ende (Fn. 30).

5 Vgl. Christian Katzenmeier, Der Behandlungsvertrag - Neuer Vertragstypus im BGB, NJW 2013, 817-823.

$6 \mathrm{Vgl}$. gegen eine analoge Anwendung der werkvertragsrechtlichen Selbstvornahmeregeln im Kauf - trotz der Annäherung des Allgemeinen Schuldrechts insgesamt an werkvertragliche Strukturen - BGH NJW 2006, 1195 (1197).

7 Vgl. dazu skizzenhaft Christian Baldus, Biens et services, goods and services: analytisch, systematisch oder gar nichts?, in: GPR 5 (2008) 1, und jetzt näher Saskia Kümmerle, „Güter und Dienstleistungen“ - Vertragstypenbildung durch den EuGH, in: Francisco Javier Andrés Santos / Christian Baldus / Helge Dedek (Hrsg.), Vertragstypen in Europa. Historische Entwicklung und europäische Perspektiven (München 2011) 295-348; Hannes Wais, Der Europäische Erfüllungsgerichtsstand für Dienstleistungsverträge. Zur Auslegung des Art. 5 Nr. 1 lit. b 2. Spiegelstrich EuGVVO (Tübingen 2013), im Erscheinen: etwa in Kap. 4.C. 
des projektierten Gemeinsamen Europäischen Kaufrechts, ${ }^{8}$ andererseits auf öffentlich-rechtlich konnotierte Probleme wie das der Zuständigkeit oder der Handlungsform. Hingegen steht die genuin zivilrechtliche und zivilrechtsvergleichende Grundsatzfrage, von welchen Typenvorstellungen wir eigentlich ausgehen wollen, nicht im Vordergrund. Um nur ein Beispiel zu nennen: Soll man den Kaufvertrag wirklich für das Paradigma des Schuldvertrages halten?

Das ist erstaunlich, betrachtet man etwa das gewaltige im Gemeinsamen Referenzrahmen zusammengetragene Material (Kern nimmt es zu seinen Themen mit der gebotenen Vorsicht in den Blick). Der DCFR liefert aber auch einen Schlüssel zu denkbaren Erklärungen: Rechtsvergleichung, die akzeptanzfähige Ergebnisse herbeiführen will, muss alle wichtigen Rechtsordnungen des ins Auge gefassten Bezugssystems in den Blick nehmen, und der DCFR kann nicht verleugnen, dass er bestimmte romanische Rechte hintan- oder doch in bestimmte Perspektiven stellt. Ebenso wird man heute nicht mehr unhinterfragt dem Mantra von der rein funktionalistischen Rechtsvergleichung folgen, riskiert diese doch - wo nicht auf hohem Niveau betrieben - zu einer Art Paradogmatik ohne Kontakt zu Wurzeln und Tendenzen zu denaturieren. ${ }^{9}$ Vielmehr gibt es, gerade aus den letzten Jahrzehnten, zahlreiche neue Erkenntnisse rechtsgeschichtlicher Art, die zu bedenken auch für Zwecke einer praktischen Komparatistik kein Fehler wäre; das gilt namentlich für die italienische und spanische Literatur zur römischen Obligationensystematik. ${ }^{10}$

Man mag angesichts dieses Bildes bedauern, dass fürs Erste keine typensensible Gesamtsystematik aus Brüssel und Straßburg zu erwarten ist. Man mag auch die Risiken einer faktischen Bildung isolierter Typen in Gesetzgebung und Rechtsprechung betonen, im Fall der europäischen Rechtsprechung weiter kompliziert durch das $\mathrm{Zu}$ sammenspiel verschiedener Spruchkörper in unterschiedlichen juristischen Zusammenhängen. Ebenso skeptisch darf man aber sein, was die derzeitige Fähigkeit des europäischen Gesetzgebers angeht, hinreichend fundierte, praktikable und damit akzeptanzfähige Normtexte zu schaffen. Die Gründe sind bekannt.

$8 \mathrm{Zu}$ diesem Text vgl. nur aus jüngster Zeit und ohne Anspruch auf Vollständigkeit - jeweils m.w.N. die Beiträge in AcP 212 (2012) 469-852; Nils Jansen u.a. (Hrsg.), Der Entwurf für ein optionales europäisches Kaufrecht (München 2012); Martin Schmidt-Kessel (Hrsg.), Ein einheitliches europäisches Kaufrecht? Eine Analyse des Vorschlags der Kommission (München 2012); ders. (Hrsg.), Der Entwurf für ein Gemeinsames Europäisches Kaufrecht (München 2013); Martin Gebauer (Hrsg.), Gemeinsames Europäisches Kaufrecht - Anwendungsbereich und kollisionsrechtliche Einbettung (München 2013); laufende Berichterstattung in GPR; aus der internationalen Diskussion etwa die Beiträge in Contratto e Impresa / Europa 17 (2012) 1-482.

9 Grundsätzlich zu Möglichkeiten und Grenzen des Funktionalismus jetzt zwei Heidelberger Dissertationen: Lena Kunz, Postmortale Privatautonomie und Willensvollstreckung (Jena 2014), Erster Teil, $\$ 2$ B.-D.; Benjamin Herzog, Anwendung und Auslegung von Recht in Portugal und Brasilien (i.V. für 2014) $\mathbb{\$} 1$.

10 Synthese und Kritik der bis 2008 erschienenen Arbeiten bei Javier Paricio, Contrato. La formación de un concepto (Cizur Menor 2008); dt. in Andrés Santos / Baldus / Dedek, Vertragstypen (Fn. 7) 12-39, 41-44. Seither etwa Luigi Capogrossi Colognesi / Maria Floriana Cursi (a cura di), Obligatio-obbligazione. Un confronto interdisciplinare (Napoli 2011). 
Vielleicht ist es daher doch besser, die Typen vorerst so weiter wachsen zu lassen, wie sie immer schon gewachsen sind: als standardisierte Antworten auf Bedürfnisse der Praxis. Wenn es einmal zu einer Europäisierung kommt, müsste der Gesetzgeber freilich sehr gründlich auf die verschiedenen Rechtserfahrungen Europas schauen; ${ }^{11}$ und er müsste den Mut haben, solche Regeln zu ändern, die sich in punktueller Gesetzgebung oder Rechtsprechung als kontraproduktiv oder systemwidrig erwiesen haben.

Wie all dem auch sei: Typenbildung im Schuldrecht wird national wie europäisch zwar in ihren Anwendungsproblemen gesehen, aber selten als Grundsatzfrage angegangen. Man schafft neue oder variierte Typen, wo die Rechtsordnung Typen als Grundlage der Rechtsanwendung wünscht, und unterlässt es, wo sie auch in einem sehr freien Umgang der Rechtsprechung mit bestehenden Normen kein Problem sieht. Ein tragfähiger (gar europäischer) Konsens ist nicht erkennbar.

5. Im Sachenrecht verhält es sich noch eigenartiger. Man kann sich der Erkenntnis nicht entziehen, dass vor allem die Kreditsicherungspraxis Institute hervorbringt, die sich schon seit langem am Rande des ausdrücklich gesetzlich Vertypten bewegen. Auf verschiedenen Wegen bringt man diese Erkenntnis in Übereinstimmung mit der herrschenden Rechtsquellenlehre und hat sich jedenfalls darauf geeinigt, dass das alles kein Problem sei; entsprechend fordert auch kaum jemand ernstlich die Aufgabe des sachenrechtlichen Typenzwangs - und in der Tat würde die Praxis sich für solche Veränderungen gewiss bedanken. (Das hat immerhin den angenehmen Nebeneffekt, dass die Diskussion um ein Schuld- und Sachenrecht überwölbendes, also historisch und systematisch denaturierendes, allgemeines Vermögensrecht derzeit gleichermaBen ruht.) Auch hier spielt Europa herein, am Horizont etwa in Gestalt der Diskussion zur so genannten Eurohypothek (dazu Kern S. 139).

6. Ebenfalls europäisch (und aus sachenrechtlicher Sicht immer noch unterschätzt) ist der Einfluss der Erbrechtsverordnung. ${ }^{12}$ Hier geht es, mit Kern (etwa S. 235-239; nicht zur VO) zu sprechen, um das Verhältnis von Typizität und Publizität: Nach zähem Ringen ist ein Formelkompromiss in der Frage gefunden worden, wie mit von Todes wegen erworbenen ausländischen Sachenrechtspositionen umzugehen sei. EGrd. 15 der Verordnung garantiert zwar ausdrücklich den jeweiligen nationalen numerus clausus; diese Garantie ist mit einer ausdrücklichen Anpassungsvorschrift

11 Einige Gedanken in Andrés Santos / Baldus / Dedek, Vertragstypen (Fn. 7).

12 VO (EU) 650/2012 des Europäischen Parlaments und des Rates vom 4.7.2012, ABl. L 201/107 v. 27.7.2012; Korrektur zu Art. 83 III: vgl. IPRax 2013, H. 4, II. Aus der Einführungsliteratur: Lena Kunz, Die neue Europäische Erbrechtsverordnung - ein Überblick, in: GPR 2012, 208-212, 253-257; ausführlich: Andrea Bonomi / Patrick Wautelet, Le droit européen des successions. Commentaire du Règlement n650/2012 du 4 juillet 2012 (Bruxelles 2013). 
abgesichert (Art. 31, ${ }^{13}$ vgl. EGrd. 16f. und EGrd. 18f. zum Registerrecht). Die Eintragung und deren Wirkungen werden ausdrücklich nicht geregelt, Art. 1 (2) lit. l) ErbVO. Die lex successionis soll an den Grenzen der lex rei sitae enden, und diese Begrenzung sollte durch den Rekurs auf die Typizität verschlüsselt werden: so - in der Tendenz gegen den ursprünglichen Kommissionsentwurf - die von Parlament und Rat beschlossene Fassung.

Freilich ist nach den am Ende beschlossenen Formulierungen durchaus unklar, wo die Grenze genau verläuft. Dazu muss man nach den Funktionen der Publizität fragen. Denn wenn jemand nach anwendbarer ausländischer lex successionis beispielsweise Vindikationslegatar ${ }^{14}$ und damit Eigentümer ist (von exotischeren Rechtspositionen hier zu schweigen), ${ }^{15}$ dann kann mit all den Verfahrens- und Typenschutznormen nicht gemeint sein, dass er doch nur eine schuldrechtliche Position innehabe. Die Verordnung hütet sich, Entsprechendes anzudeuten. Ganz im Gegenteil sagt EGrd. 42 in konsequenter Umsetzung der Gesamtkonzeption: „Das zur Anwendung berufene Erbrecht sollte für die Rechtsnachfolge von Todes wegen vom Eintritt des Erbfalls bis zum Übergang des Eigentums an den zum Nachlass gehörenden Vermögenswerten auf die nach diesem Recht bestimmten Berechtigten gelten. (...)“

Aufschlussreich sind auch EGrd. 67f. zum Europäischen Nachlasszeugnis: Der Zugang zum ausländischen Register soll einfach sein, und die „das Zeugnis ausstellende Behörde sollte die Formalitäten beachten, die für die Eintragung von unbeweglichen Sachen in dem Mitgliedstaat, in dem das Register geführt wird, vorgeschrieben sind “. „Formalitäten“ heißt nicht Marginalien, Praktikabilität und Rechtssicherheit müssen gewahrt bleiben - aber eben auch das Recht des Berechtigten. ${ }^{16}$ Entsprechend ordnet Art. 23 (2) lit. e) unter anderem an, dem Erbstatut unterliege „der Übergang der zum Nachlass gehörenden Vermögenswerte, Rechte und Pflichten auf die Erben und gegebenenfalls die Vermächtnisnehmer (... “; in dieselbe Richtung weist u.a.

13 „Anpassung dinglicher Rechte

Macht eine Person ein dingliches Recht geltend, das ihr nach dem auf die Rechtsnachfolge von Todes wegen anzuwendenden Recht zusteht, und kennt das Recht des Mitgliedstaats, in dem das Recht geltend gemacht wird, das betreffende dingliche Recht nicht, so ist dieses Recht soweit erforderlich und möglich an das in der Rechtsordnung dieses Mitgliedstaats am ehesten vergleichbare Recht anzupassen, wobei die mit dem besagten dinglichen Recht verfolgten Ziele und Interessen und die mit ihm verbundenen Wirkungen zu berücksichtigen sind.“.

14 Grundsätzlich zum Vindikationslegat jetzt Jan Peter Schmidt, Die kollisionsrechtliche Behandlung dinglich wirkender Vermächtnisse. Ein Prüfstein für Grundsatzfragen des internationalen und des materiellen Privatrechts, in: RabelsZ 77 (2013) 1-30. Die von Schmidt richtig betonten Wertungsgesichtspunkte laden zu weiterer historischer und vergleichender Vertiefung ein.

15 Solche bieten etwa die spanischen Foralrechte; vgl. nur Robert Beier, Die gesetzlichen Rechte des überlebenden Ehegatten nach dem deutschen und spanischen Kollisionsrecht. Unter besonderer Berücksichtigung der viudedad aragonesa (Frankfurt a.M. u.a. 2009). Aber auch die gemeinspanischen dinglich wirksamen Teilungsbefugnisse des Testamentsvollstreckers können Probleme bereiten; s. Lena Kunz, Nachlassspaltung durch die registerrechtliche Hintertür. Zur Koordination des Erb-, Sachund Registerstatuts in der EuErbVO, in: GPR 2013, 293ff.

16 Was sich die VO unter den diversen Berechtigten vorstellt, ist freilich nicht ganz klar, vgl. Christian Baldus, Erbe und Vermächtnisnehmer nach der Erbrechtsverordnung, in: GPR 2012, 312-315. 
lit. j) derselben Norm zur Teilung des Nachlasses. Art. 69 (5) schließlich stellt zum Nachlasszeugnis klar: „Das Zeugnis stellt ein wirksames Schriftstück für die Eintragung des Nachlassvermögens in das einschlägige Register eines Mitgliedstaats dar, unbeschadet des Artikels 1 Absatz 2 Buchstaben k und 1.“

Die Formelkompromisse, ${ }^{17}$ anders gesagt: die inneren Spannungen, sind offenkundig. In solcher Lage entscheidet das télos. ${ }^{18}$ Danach dominiert, im Sinne der Privatrechtsintegration und des Zugangs zum Recht, die lex successionis - soweit mit funktionalen Anforderungen des Registerrechts vereinbar. Darauf wird das Sachenrecht der Mitgliedstaaten sich einstellen müssen. ${ }^{19}$

Praktisch stellt sich also die Frage, wie die existierende ausländische Position in das deutsche Register kommt. Hier bedarf es keiner großen Phantasie, eines vorauszusagen: Nationale Verfahren, welche die (EU-)ausländische Position de facto zu einer schuldrechtlichen entwerten, wird der EuGH nicht akzeptieren, und ebenso wenig solche, die ausländischen Erbnachweisen ohne zwingenden Grund weniger Kraft geben als inländischen. Denn die justizielle Zusammenarbeit soll dem Bürger effektiv zu Gute kommen. Wann der erste Vorlagebeschluss nach Wirksamwerden der Verordnung ergehen wird, ist nur eine Frage der Zeit. Eine Autonomie von Verfahren und Register, die im Ergebnis Grundentscheidungen der Verordnung aufhöbe, wird Luxemburg nicht akzeptieren ${ }^{20}$ - schon gar nicht dann, wenn nicht das praktische Bedürfnis für den Schutz bestehender registerrechtlicher Strukturen eindeutig dargetan wird, und zwar hinsichtlich konkreter Anwendungsprobleme. Die Debatte über dieses Bedürfnis lässt sich noch wesentlich vertiefen; mit Erlass der Verordnung scheint das Interesse aber zuzunehmen.

Inwieweit mit alldem wirklich Fragen der Typenbildung betroffen sind, ist mithin bislang ungeklärt. Der erwähnte Vorbehalt zugunsten des nationalen numerus clausus jedenfalls löst das Problem wohl nicht. Das lässt sich auch allgemeiner fassen: Unionsrecht folgt nicht nationalen oder gar regionalen Schemata und Typologien, sondern erwartet funktions- und europakonforme Ausgestaltung der mitgliedstaatlichen Rechte. Damit können diese durchaus leben, und hier sind wir schon bei einer zentralen These des zu besprechenden Werkes: der weitgehenden Funktionsgerechtigkeit sachenrechtlicher Typenbildung in Deutschland (u. 9.). Was übrigens das

17 Hier ist aus Raumgründen nur die deutsche Fassung zitiert; konzipiert wurde die Verordnung vorwiegend auf Französisch, im Rat verhandelt vorwiegend auf Englisch. Zu methodologischen Konsequenzen demnächst Christian Baldus / Thomas Raff, $\mathbb{\$}$ 3: Richterliche Interpretation des Gemeinschaftsrechts, in: Enzyklopädie Europarecht, Band 6: Europäisches Privat- und Unternehmensrecht (hrsg. v. Martin Gebauer und Christoph Teichmann), Baden-Baden 2014.

18 Zum Problem mwN. Baldus / Raff (wie vor).

19 Vgl. bereits zu einer der Entwurfsfassungen Christian Baldus, Le temps de l'exploration, in : L'avenir européen du droit des successions internationales. Sous la dir. de Hubert Bosse-Platière, Nicolas Damas et Yves Dereu (Paris 2011) 85-94.

20 Zum Problem jetzt Kunz (Fn. 15). Dagegen demnächst Christoph Döbereiner, in: GPR 2014. 
Verhältnis von Typizität und Publizität angeht, sagt Kern im historischen Teil, dass sie oft zusammenfielen, weil erstere letztere natürlich erleichtere, dass aber bei voller Information Typizität nicht zwingend nötig sei (S. 236). In der abschließenden Bewertung qualifiziert er dasjenige Maß an schuldrechtlicher Öffnung, das nach deutschem Recht neben den bewährten sachenrechtlichen Figuren möglich ist, als angemessen (S. 520-523). Für die verschiedenen Spielarten ausländischer dinglicher Berechtigungen müsste man entsprechende Fragen stellen.

7. Vermerkt sei noch, dass im Familienrecht eine interessante Sonderentwicklung zu beobachten ist: das Streben nach dem Typus sozusagen um seines Namens willen, mindestens so sehr wie wegen seiner Inhalte. ${ }^{21}$ Dass familienrechtliche Statusfragen definiert sein müssen, wird kaum in Frage gestellt. Der gesellschaftliche Veränderungsdruck geht aber nicht mehr, wie in den siebziger und achtziger Jahren, auf Abschleifung eines als bürgerlich empfundenen Instituts, der Ehe, sondern auf Öffnung eben dieses Instituts für bislang ausgeschlossene Lebensformen. Der deutsche Gesetzgeber bietet in Gestalt der eingetragenen Lebenspartnerschaft ein Ersatzinstitut an, das der Laie schon sprachlich kaum von nicht geregelten Formen des Zusammenlebens unterscheiden kann, die Rechtsprechung arbeitet auf eine Gleichbehandlung mit der Ehe hin; parallel gibt das Internationale Privatrecht einzelnen Interessenten die Möglichkeit, ausländische Status zu erlangen, die im Ausland Ehe heißen. So wird das an sich scharf definierte Institut der Ehe in der Sache aufgelöst: sozusagen typisiert? Auch her zeigt sich jedenfalls, dass im Familienrecht die Uhren anders gehen als im Rest der Kodifikation, jedenfalls und soweit es Statusrecht ist.

8. Die Typenproblematik, so lässt sich resümieren, stellt sich für die traditionellen Teilgebiete des allgemeinen Privatrechts jeweils unterschiedlich, und sie ist sperrig. Angesichts dieses Bildes gehört ein gewisser Mut dazu, sich ihrer in einer Habilitationsschrift anzunehmen. Einerseits gilt sie nicht als aktuell, andererseits nicht als lösbar; man kann mit ihrer zerfaserten Ungelöstheit leben. Hieraus folgt, wie der Ansatz eines doch zu schreibenden Buches aussehen musste: rechtsvergleichend, nicht ohne einerseits geschichtliche, andererseits theoretische Grundierung, und gerichtet darauf, einer solcherart umfassend erhobenen Praxis ein systematisch überzeugendes, also anwendungsfähiges Theorieangebot zu machen. Dies ist Christoph Kern in glänzender Weise gelungen.

Der fünfteilige Aufbau ist eingängig: Nach konziser Einführung sichert ein Grundlagenkapitel (S. 6-33) gegen begriffliche Befangenheiten rund um den Typus. Es folgen zweiter (Sachenrecht, S. 35-251) und dritter Teil (Wertpapierrecht, S. 253-449): die Befunde zu den ausgewählten Referenzgebieten jeweils in Deutschland, Frankreich, England und den Vereinigten Staaten. Sodann werden die Erkenntnisse im

21 Zu dieser „Umcodierung der Ehe“ zuletzt aus nichtjuristischer Sicht Michael Sievernich, Stimmen der Zeit 2013, 649f. 
vierten Teil gebündelt und formuliert (S. 451-544, bescheiden schreibt Kern „Erklärungsversuche, Bewertung und Ausblick“), im fünften wiederum konzis resümiert (S. 545-550; am besten gleich nach der Einleitung zu lesen). Klare Zusammenfassungen, meist mit Würdigung, bisweilen mit Prognose, sowie ein hilfreiches Sachregister erschließen effizient auch die einzelnen Kapitel. Die stets gepflegte und angemessene Sprache rundet den Eindruck ab.

9. Schon das Grundlagenkapitel zeichnet sich durch Selbständigkeit und Vorsicht aus (s. nur S.12-18 zur Typenfixierung, S. 27-33 zur begrenzten Bedeutung der deutschen Theoriediskussion, aber auch der historischen Belastung des Typusbegriffs).

Bemerkenswert ist im sachenrechtlichen Teil vor allem der Befund, dass - trotz des bekannten Gefälles zum civil law - die Typizität auch in den beiden untersuchten common law-Rechtsordnungen zunehme. Das spricht für die Existenz sachlicher Gründe für Typizität und für die Grenzen eines allein Fragen des Informationszugangs privilegierenden Ansatzes. Um gleich die „Ergebnisse und Zusammenhänge“ (S. 228-251) und hier vor allem die „Regelmäßigkeiten der Entwicklung“ (ab S. 241) anzusprechen: Rechtshistoriker sind häufig durch die von der Geschichtswissenschaft ausstrahlende Methodendiskussion übervorsichtig gegenüber der Frage nach solchen Strukturen geworden. Begreift man aber Rechtsgeschichte als normativ informierte Untersuchung der Kontinuität und Diskontinuität von Problemen und Lösungen, ${ }^{22}$ dann muss man sich freuen, wenn ein historisch informierter Dogmatiker genau so fragt und antwortet wie Kern. Am Ende wollen wir als Juristen ja etwas lernen und wissen auch genau, dass das durchaus geht.

Kern formuliert klar und abgewogen: Es gebe im Sachenrecht historisch-vergleichend weder völlige Gestaltungsfreiheit noch strengste Typizität; die Typizität nehme tendenziell überall zu, auch in den Ländern des common law; in diesen solle sie noch weiter zunehmen, wohingegen die kontinentalen Rechtsordnungen eher einer „Vergewisserung über die Hintergründe des erreichten Standes“ (S. 251) bedürften; das „Störpotential“ grenzüberschreitender Sachverhalte halte sich in Grenzen (zum Unionsrecht sieht Kern freilich berechtigterweise Entwicklungsmöglichkeiten, S. 232 vgl. auch das oben zum Erbrecht Gesagte). Näher ausgeführt wird, dass der genaue Zusammenhang zwischen Typizität und Publizität alles andere als einheitlich sei (S. 235-239): Höhere Typizität begegne in historisch-vergleichender Perspektive vor

$22 \mathrm{Zu}$ theoretischen Fragen aus der Sicht des Unterzeichneten vgl. Christian Baldus, Warum Exegese? Einige Thesen zur Methode der privatrechtlichen Romanistik, in: Wactaw Uruszczak / Paulina Swiecicka / Andrzej Kremer (eds.), Leges sapere. Studia i prace dedykowane Profesorowi Januszowi Sondlowi w piecdziesiata rocznice pracy naukowej (Studies and Essays dedicated to Professor Janusz Sondel on his 50th anniversary of scientific work) (Kraków 2008) 87-101; ders., Verfahren, Wahnsinn und Methode. Modestin D. 27,8,27pr. als Element einer politischen Methodengeschichte der Jurisprudenz, in: Arndt Kiehnle / Bernd Mertens / Gottfried Schiemann (Hrsg.), Festschrift für Jan Schröder zum 70. Geburtstag am 28. Mai 2013 (Tübingen 2013) 3-19 (17ff.). 
allem bei (wie Kern formuliert) transaktionsbezogener Publizität, also konstitutiven Publizitätsakten. Die erste Kernthese zu regelmäßigen Entwicklungen lautet dann, Typizität wachse mit dem Kreis potentieller Berechtigter wie mit dem potentieller Objekte (letzteres vor allem bei Grundstücken). Das wird auch wirtschaftsgeschichtlich nochmals untermauert.

Verhält es sich so, dann hat die Typizität in der Tat Zukunft auch in der Union. Die Union bedarf gerade dann aber auch einer intensiveren rechtsvergleichenden Debatte über die denkbaren Typen. Eine solche Debatte sollte ihren Schwerpunkt (noch) nicht unbedingt bei mehr oder minder fundierten restatements haben, auch wenn diese im Vorfeld gesetzgeberischen Tätigwerdens nützlich sein können. Sie sollte zunächst in präzisen Einzelstudien zum Funktionieren bestimmter Institute in bestimmten Ländern weitere Fundamente legen: über wichtige praktische Details hinaus eben die zu Recht angemahnte „Vergewisserung“. Kerns Werk kann man jedem Doktoranden, der eine solche Studie angeht, als Ausgangspunkt und Grundlage ans Herz legen.

Das Wertpapierrecht konnte Verf. nicht mit parallelem Aufbau angehen, schon weil die Wurzeln des Gebietes jünger sind, es also an einheitlicher antiker Prägung mangeln muss. Er stellt folglich funktionell bestimmte Gruppen vor: Wertpapiere des Kredit- und Zahlungsverkehrs, des Warenverkehrs und - hier die jüngsten Entwicklungen - des Kapitalmarkts. Die Ergebnisse und Zusammenhänge (S. 431-449) divergieren in einigen Punkten vom Sachenrecht und liefern eben dadurch eine gute Grundlage für den abschließenden Vergleich: Im Wertpapierrecht seien die Divergenzen im Grad der Typisierung zwischen Wertpapieren, aber auch zwischen Rechtsordnungen und -traditionen (civil law / common law) deutlicher, und (natürlich) spiele der internationale Verkehr eine größere Rolle. Was die Zunahme an Typizität angeht, vermag Kern nur für die Wertpapiere des Kredit-, Zahlungs- und Warenverkehrs eine der sachenrechtlichen ungefähr parallele Entwicklung auszumachen; ganz anders im Kapitalmarktrecht, wo teilweise „jede verlässliche Typizität“ fehle (S. 437). Für die kapitalmarktrechtliche Publizität spiele Typizität eine lediglich untergeordnete Rolle. Soweit - in partieller Parallele zum Sachenrecht - Erweiterungen des betroffenen Personenkreises und Gegenstandsbereichs zu verzeichnen seien, hätten diese wiederum nur im Kredit-, Zahlungs- und Warenverkehr die Typizität gesteigert. Dass die typizitätsfeindliche Entwicklung im Kapitalmarktrecht von Dauer sei, scheint Verf. unwahrscheinlich (S. 449), und an diesem Punkt kann er zu strukturellen Folgerungen übergehen.

Bei den Erklärungsversuchen (S. 452-507) geht er zunächst auf neoklassische ökonomische Ansätze ein (über jene Grundzüge hinaus, die bisweilen pompöse Untertitel zur „ökonomischen Analyse“ rechtfertigen sollen) und arbeitet heraus, dass diese Ansätze es sich jedenfalls zu einfach machen. Der hingegen gebotene mehrdimensionale Ansatz verbinde Transaktionskostenökonomik mit juristischen Erfahrungs- 
werten. Kern spricht sogleich (S. 463) den Prüfstein für die Richtigkeit dieses Ansatzes an: Er muss auch das strukturell abweichende Bild im Kapitalmarktrecht erklären können. Das wird durchgespielt für die Parameter Qualität der angebotenen Typen, Reduzierung von Informationskosten, Kanalisierung auf bestimmte Gestaltungen (mit dem verwandten, schon zuvor durchgängig geprüften Aspekt der Missbilligung bestimmter Gestaltungen). Ergebnis in der Tat: „Eigene Wege gehen hingegen die Kapitalmarktpapiere“ (S. 483; vgl. S. 490 und sqq.). Das wird näher ausgeführt, nicht ohne Warnung davor, die Mechanismen zu überschätzen, die auf dem Kapitalmarkt Typizität ersetzen sollen (S. 507). Für die anderen Untersuchungsobjekte sieht Kern die Parameter bestätigt. Er weist sehr zu Recht allerdings auch nochmals darauf hin, dass nach aller Erfahrung erleichterte Informationsmöglichkeit allein Typizität nicht verdrängt (S. 489). Das wird man bei registerfähigen Gütern im Auge haben müssen, selbst bei optimal angelegten Registern (auch und gerade im oben erörterten Überschneidungsbereich Erbrecht/Sachenrecht).

Die Bewertung fällt eindeutig aus: Typizität sei ein Strukturprinzip übertragbarer Güter (ab S. 508). Dies werde im Ergebnis durch die mit ihr verbundenen Beschränkungen privatautonomen Handelns weder praktisch noch theoretisch in Frage gestellt, solange die Rechtsordnung den Parteien gebe, was sie bräuchten; dies sei in den europäischen Sachenrechten und namentlich im deutschen geradezu „musterhaft" (S. 518) realisiert. Im Wertpapierrecht bleibt Kern bei seiner kritischen Bewertung der als defizitär gekennzeichneten kapitalmarktrechtlichen Typizität, schlägt aber (realistischerweise) nur einen schrittweisen Ausbau typisierter Formen vor (S. 536f.)

Nach diesem (sehr summarischen) Abriss: Hier kann der Leser weiterfragen. Was sind die spezifisch grundlagen- und systembezogenen Aspekte, also das, was eine umfassend wissenschaftlich ${ }^{23}$ konzipierte Typizitäts- oder auch Typenlehre gerade ausmachen kann? Die Frage drängt sich umso mehr auf, als man Kerns auch historische Aufarbeitung auf die beiden eingangs angesprochenen Gesichter des Typus beziehen könnte. Wie belastbar bleiben gesetzliche Typen in dem, was man wohl den Regelbereich wird nennen können, also in den Feldern steigender Typizität? Unter welchen Bedingungen lässt sich hingegen im Kapitalmarktbereich auf spontane Verdichtung der unübersichtlichen Strukturen hoffen?

10. Kern widmet der Rechtsgeschichte breiten Raum und kommt zu Ergebnissen, die dem Rechtshistoriker wie dem Dogmatiker Respekt einflößen. Dabei geht es weniger um die neuere Rechtsgeschichte, namentlich den massiven Gebrauch des Typuskon-

23 Jedenfalls insoweit ist dem Wissenschaftsrat beizupflichten, s. http://www.wissenschaftsrat.de/download/archiv/2558-12.pdf. Näher Christian Baldus, Anuario de Derecho Civil 66 (2013) 5-26. 
zepts im Nationalsozialismus: ${ }^{24}$ Diese Fragen sind mittlerweile recht gut aufgearbeitet und auch über den Kreis der Spezialisten hinaus bekannt; dass jüngere Autoren fröhlich Larenz-Schriften aus den 1940er Jahren zum Beleg eines als unproblematisch empfundenen Typusbegriffs zitieren, wird seltener. Hierzu geht Kern gleich eingangs einen soliden und nachdenklichen Weg: Er sagt am Ende seiner begrifflichen Grundlegung, dass die Typusdebatte auch aus auslegungspraktischen Gründen keine große Rolle spiele und dass unter der jeweiligen Methode der Rechtsfindung zu fragen sei, warum und mit welchen Ergebnissen es zur Vereinheitlichung komme (S. 32). Er funktionalisiert damit legitimerweise den Typusbegriff, anstatt sich zum Gefangenen rechtstheoretischer Festlegungen zu machen. Für sein Thema ist dies schon deswegen sinnvoll, weil in Deutschland unter totalitärer Herrschaft die sachenrechtliche Typizität gelitten hat, nicht zuletzt unter der zeitgenössischen Typenlehre (S. 134).

Sogleich im Anschluss wendet sich Kern näher dem römischen Recht zu, dem bekanntlich große Teile des geltenden (nicht nur deutschen) Sachenrechts entstammen. Hier nun stellt sich eine Grundsatzfrage: Finden wir in der römischen Kasuistik das heutige Phänomen des Typus als einer scharfe Subsumtion abmildernden oder vermeidenden Figur? Insoweit nicht, so ist zunächst festzuhalten, als das Gesetz in Rom nicht so dominierte wie heute ${ }^{25}$ und als die rechtsschöpferische Aufgabe der Juristen unbestritten war. Sie hatten so viel Freiheit, dass sie keines Weichspülers bedurften, und einen „Positivismus“ oder „Formalismus“, wie ihn die Typusideologen des 20. Jahrhunderts in polemischer Absicht dem 19. Jahrhundert zuschrieben, ${ }^{26}$ gab es schon gar nicht. Vielmehr entstanden die meisten Strukturen heutigen Privatrechts gerade im inneren System der an typisierten actiones orientierten römischen Gutachtenpraxis. Dort finden wir sowohl scharfe Subsumtion als auch offene Wertung im Einzelfall, mit allen denkbaren Übergängen und Verbindungen. Umstritten ist freilich (schon angesichts der Lakonik, die viele Quellen kennzeichnet), ${ }^{27}$ ob diese

24 Grundlegend außer den bekannten Studien von Rüthers (s. nur Bernd Rüthers / Christian Fischer / Axel Birk, Rechtstheorie mit Juristischer Methodenlehre, 6. Aufl. München 2011 mwN.) Josef Kokert, Der Begriff des Typus bei Karl Larenz (Berlin 1995). Zur verwandten Problematik der Generalklauseln ältere Lit. bei Christian Baldus / Peter-Christian Müller-Graff (Hrsg.), Die Generalklausel im Europäischen Privatrecht. Zur Leistungsfähigkeit der deutschen Wissenschaft aus romanischer Perspektive (München 2006); zuletzt Bernd Mertens, Das Konzept der Generalklauseln im Allgemeinen Teil des BGB, in: Wojciech Dajczak / Christian Baldus (Hrsg.), Der Allgemeine Teil des Privatrechts. Erfahrungen und Perspektiven zwischen Deutschland, Polen und den lusitanischen Rechten (Frankfurt a.M. u.a. 2013) 99-119 mwN. $111 \mathrm{ff}$.

25 Dazu jetzt umfassend Jean-Louis Ferrary (a cura di), Leges publicae. La legge nell'esperienza giuridica romana (Pavia 2012), mit Besprechung von Christian Baldus in: Der Staat 52 (2013) 331-335.

26 Hierzu zuletzt Hans-Peter Haferkamp, Zur Methodengeschichte unter dem BGB in fünf Systemen (Vortrag auf der Zivilrechtslehrertagung 2013, vorgesehen für AcP 2014).

27 In dieser Lakonik und Praxisorientierung liegt ein wesentlicher Grund dafür, dass römischrechtliche Forschung exegetisch betrieben sein will: Entscheidungstendenzen und erst recht "allgemeine Grundsätze" gibt die typische Quelle nur bei genauer Sachverhalts- und Kontextanalyse preis. Vgl. nochmals Baldus, Warum Exegese? (Fn. 22). 
Polarität zwischen Subsumtion und Wertung sich auf eine grundsätzliche Matrix zurückführen lässt. Dies behaupten einige und sehen philosophische Einflüsse am Werk; ${ }^{28}$ die herrschende Auffassung ist vorsichtiger und nimmt eher thematisch bedingte Differenzierungen sowie individuelle Juristenstile an. ${ }^{29}$

Das ist vermintes Terrain, auf das reine Geltendrechtler sich vorsichtigerweise zumeist nicht begeben. Kern kennt die Problematik und optiert für einen pandektistisch-materiellrechtlichem Denken zugänglichen, aber historisch-prozessual informierten Aufbau: $\mathrm{Zu}$ den großen Epochen des römischen Rechts spricht er zunächst die Typen und Gestaltungsmöglichkeiten an, dann unter „Rechtliches Umfeld“ namentlich den Prozess. (Auch im Weiteren wird er jeweils fragen, wie prozessual gewachsene Typenbildung fortwirkte, so etwa S. 202, 208 zu England.) Er folgt dem romanistischen Hauptstrom, vermeidet Untiefen und arbeitet heraus, dass die Typenfixierung des klassischen Rechts vor allem auf dem prätorischen Edikt beruhte und dass sie als weithin sachgerecht akzeptiert wurde; weiterhin, dass die justinianische Kompilation die Typizität gegenüber nachklassischen Entwicklungen wieder stärkte. Mit diesen Ausführungen ist die Grundlage für die zahlreichen historischen Aussagen in den Folgekapiteln gelegt; Kern kann für andere Zeiten und Räume stets dartun, welche Einflüsse warum Bedeutung erlangten.

Ihn interessiert vor allem, was man über die Herkunft der einzelnen sachenrechtlichen Typen sagen kann. Hier kommt man zu den actiones, nach denen das römische Recht - als prozessual geprägte Ordnung - strukturiert ist. Der Leser wird wiederum gut informiert, und auf dieser Grundlage baut das restliche Werk dann in gleicher Qualität auf. Wir können hier nicht alle Epochen und Traditionen Revue passieren lassen; die Ergebnisse wurden bereits skizziert. Jedenfalls lässt sein Werk sich zugleich lesen als Bestandsaufnahme für eine vergleichende Geschichte des Eigentums in Europa.

11. Zur systematischen Hinsicht wurden Ergebnisse bereits referiert. Unterstrichen sei: Kern gibt weder vor, systemunabhängig zu denken (das wäre eine Illusion überzogenen Funktionalismus', man kann und muss es nur versuchen), noch ist er einem bestimmten System verhaftet. Dass er am Ende Strukturen des deutschen Rechts positiv bewertet, beruht nicht auf Vorurteilen, sondern auf einer umfassenden Untersuchung, die sich auf französische und angloamerikanische Modelle ernsthaft eingelassen hat. Der vielleicht wichtigste Beleg für diese Sensibilität sind Kerns Vorbehalte zum Unionsprivatrecht, einem Gebiet, in dem der Autor gleichfalls gut ausge-

28 So namentlich Okko Behrends, Institutionelles und prinzipielles Denken im römischen Privatrecht, SZ 95 (1978) 187-231 (später mehrfach fortentwickelt); kritisch etwa Jan Dirk Harke, Rezension, RabelsZ 72 (2008) 226-229.

29 Diskussionsstand: Christian Baldus / Gianni Santucci / Massimo Miglietta / Emanuele Stolfi (a cura di), Dogmengeschichte und historische Individualität der römischen Juristen. Storia dei dogmi e individualità storica dei giuristi romani, Lavis (Trento) 2012. 
wiesen ist. Die Erkenntnisse aus der Monographie versprechen gerade für dieses Gebiet reichhaltige weitere Erkenntnis. Die Forschung zur Typenbildung in Europa steht dogmatisch, historisch und vergleichend erst am Anfang.

12. Rehabilitiert Kern mit alldem den Typus, auch wenn es ihm nicht primär auf diese Begrifflichkeit ankommt? Er rehabilitiert den numerus clausus, soweit ein gewisses Maß an Beweglichkeit erhalten bleibt. Diese Figur hat nicht ausgedient, soweit sie strukturellen Interessen der Parteien (nicht punktuellen Sonderinteressen) dient. Mit dem Typus als Manipulationsinstrument hat sie nichts zu tun. Welche Begriffe wir wählen wollen, ist demnach zwar in der Tat nicht die Hauptfrage; es wäre aber gerade im Licht dieser Arbeit sinnvoll, sie zu klären: im Sinne leistungsfähiger Dogmatik.

Dogmatik, das hat jüngst wieder Rolf Stürner betont, ${ }^{30}$ ist gerade in ihren Details keine Selbstverständlichkeit, sondern typischer Ausdruck der kontinentaleuropäischen Rechtskultur: ein Instrument zu sachgerechter Falllösung, das unter heutigen Bedingungen besonders bewusst gepflegt werden muss, soll es überleben und sich weiterentwickeln. Die Missverständnisse beginnen bekanntlich schon damit, dass die Worte „Dogmatik“ und „dogmatisch “ in mehr als eine Sprache nicht oder nicht ohne negative Konnotation zu übersetzen sind.

Kerns Leistung liegt nicht zuletzt darin, für ein sehr klassisches und ein sehr dynamisches Gebiet gezeigt zu haben, was Dogmatik leisten kann: eine kulturell informierte Dogmatik, die sich gründlich über historische wie über ausländische Erfahrungen beugt, ohne dabei die Anwendungsfragen deutscher und europäischer Gegenwart je aus dem Auge zu verlieren. Dogmatik ist eben, entgegen dem Vorurteil, nicht blindes Festhalten an einmal gewonnenen Aussagen über Normauslegung, sondern anwendungsbezogene Normauslegung im Lichte aller verfügbaren Erkenntnisquellen. Kerns intelligent komponierte, jederzeit klare Darstellung zeigt, warum wir Rechtswissenschaft auch in Zukunft bewusst als dogmatische zu betreiben haben.

30 Rolf Stürner, Die Zivilrechtswissenschaft und ihre Methodik - zu anwendungsbezogen, zu wenig grundlagenorientiert? (Vortrag auf der Zivilrechtslehrertagung 2013, vorgesehen für AcP 2014). 